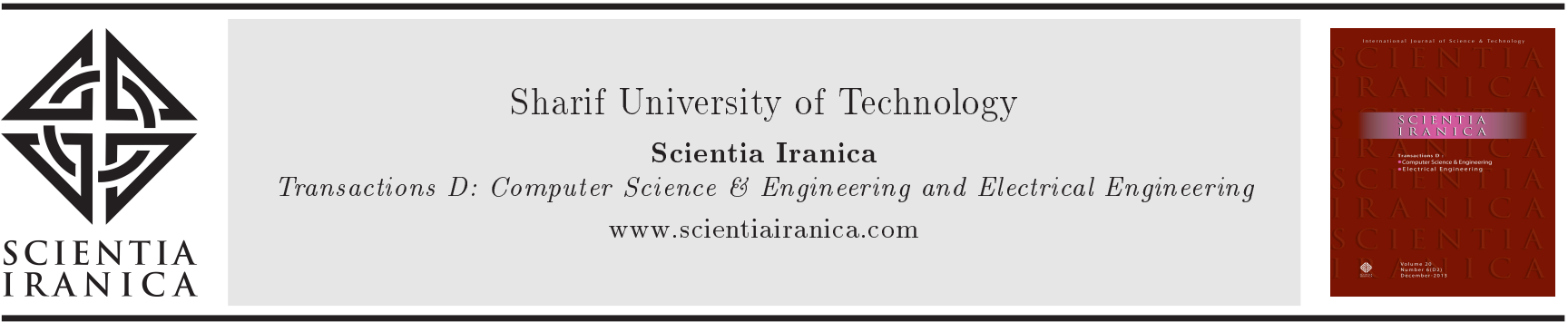

Invited Paper

\title{
Wide-band modeling of modular multilevel converters using extended-frequency dynamic phasors
}

\author{
S. Rajesvaran and S. Filizadeh* \\ Department of Electrical and Computer Engineering, University of Manitoba,75A Chancellor's Circle, Winnipeg, MB R3T 5 V6, \\ Canada.
}

Received 12 March 2016; received in revised form 20 April 2016; accepted 18 July 2016

\author{
KEYWORDS \\ Modular Multilevel \\ Converter (MMC); \\ Voltage-Source \\ Converter (VSC); \\ High-Voltage Direct \\ Current (HVDC); \\ Electromagnetic \\ transient (EMT); \\ Dynamic phasors; \\ Modeling.
}

\begin{abstract}
This paper presents a model for a Modular Multilevel Converter (MMC) using extended-frequency dynamic phasors. The model is based upon a series of harmonicfrequency representations that are obtained for the fundamental and dominant harmonic components. Depending on the requirements of the study to be conducted and the desired level of model accuracy, a low-order model (i.e., average-value) or an arbitrarily wideband model may be constructed. The paper describes the principles of modeling using extended-frequency dynamic phasors, and applies them to an MMC connected to a power system represented using a Thevenin equivalent. The model contains details of the MMC's control system including its high-level control circuitry, as well as voltage balancing and synchronization components. The developed model is then validated by comparing it against a fully-detailed electromagnetic transient (EMT) simulation model developed in PSCAD/EMTDC simulator. Comparisons are made to establish the accuracy of the model (both its low-order and wide-band variants) and to assess the computational advantages it offers compared to conventional EMT models.
\end{abstract}

(C) 2016 Sharif University of Technology. All rights reserved.

\section{Introduction}

In High-Voltage Direct Current (HVDC) transmission systems, thyristor-based Line-Commutated Converters (LCC) have been used for decades. LCC-HVDC offers the benefits of a mature technology and is available at ratings reaching thousands of MWs per converter. LCC-HVDC systems, however, suffer from technical challenges such as large filtering requirements, limited ability to operate into weak ac systems, and converter commutation failure. As such, a new breed of HVDC converters, which are based upon voltagesource converters (VSC-HVDC), have been pursued to alleviate these problems. VSCs utilize fully-controlled

*. Corresponding author. Tel.: +1-204-480-1401;

E-mail addresses: rajesvas@myumanitoba.ca (S.

Rajesvaran); shaahin.filizadeh@umanitoba.ca (S. Filizadeh) switches such as Insulated-Gate Bipolar Transistors (IGBTs) instead of thyristors to avoid commutation failure problems [1]. A class of VSC topologies, known as Modular Multilevel Converters (MMCs), has been introduced [2], which offer reduced harmonics (and hence reduced filtering requirements), lower switching losses, and superior output quality to conventional two-level and multilevel voltage-source converters. An MMC is constructed using a cascade connection of a large number of identical units known as submodules. Commonly used types include half-bridge and fullbridge submodules [1-4].

The benefits of MMC-based HVDC converters are achieved at the expense of increased control and operating complexity. MMC converters deploy a large number of submodule capacitors, whose voltages need to be tightly regulated and balanced for proper operation. Additionally, the generation of high-quality 
output voltages without incurring large switching losses requires use of advanced waveform synthesis techniques [5-7].

Similar to other high-power converter systems, many of the studies pertinent to the design, controller tuning, and operating characteristics of MMC-HVDC systems rely on detailed computer simulations. In particular, electromagnetic transient (EMT) simulators are prime simulation platforms for this purpose due to their wide-band representation of transient phenomena in switching circuits. The complexity of MMC circuitry and the presence of a large number of switches pose major computational difficulties for EMT simulations. It is often observed that simplifications need to be made in order to enable EMT simulation of MMC systems within reasonable computing time. MMC models with reduced computational intensity fall into two general categories of averaged (i.e., low-frequency) models, and low-intensity full-order (also known as detailed equivalent) models. Generally, MMC models are developed with a pre-determined modeling outcome in mind. Low-order average-value models are inherently unable to capture transients of high frequencies, and detailed models naturally include as much detail as possible within the simulation bandwidth [8].

This paper proposes a new MMC model that is based upon extended-frequency dynamic phasors. These mathematical artifacts are based upon decomposing a (quasi-) periodic waveform into constituent harmonics, which are then included in the final model. By doing so, the user of the resulting model can judiciously select the model's desired level of accuracy by including as many harmonic components as needed. This affords a high degree of control to the user to adjust the band-width of the model depending on the requirements of the study to be conducted. It is obvious to note that this modeling approach will also offer flexibility in its final computational complexity.

Modeling using dynamic phasors has been applied to such complex systems as LCC-HVDC, VSC-HVDC, and wind energy systems [9-11]. A preliminary (and only low-order) dynamic phasor model of an MMC system is presented in $[12,13]$ wherein the eigenvalues of the system are studied; the presentations in $[12,13]$, however, do not include any validation of the model. Another phasor model of an MMC is presented in [14], which is developed in a rotating d-q frame. However, this model only includes the fundamental and the second harmonic corresponding to the MMC arm's circulating current. As such, expansion of these models to include arbitrary higher-order harmonics, as is done in this paper, remains unresolved.

The extended-frequency dynamic phasor model of an MMC in this paper includes its controls and is validated against a detailed EMT model developed in PSCAD/EMTDC electromagnetic transient simulation program. The dynamic phasor model is developed directly in the original abc frame, which enables direct interfacing possibility with other system components modeled in the abc domain (e.g., in the context of EMT simulations). To facilitate the readability of the mathematical development of the model, a Nomenclature is included at the end of the paper.

\section{Mathematical principles of modeling using dynamic phasors}

Modeling using dynamic phasors is based upon decomposition of a quasi-periodic time-domain waveform into its harmonics using Fourier analysis over a sliding window. As the window slides along the time axis, the time evolution of the Fourier coefficients is obtained. For reconstruction, all or a subset of harmonics may be selected. The former produces the original waveform and the latter gives an approximation of the original waveform. The approximation can be made more accurate if a larger subset of harmonics is selected for reconstruction, albeit at the expense of more computations. The governing equations for decompositions of a signal $x(t)$ over its period $T_{0}$ are given in Eqs. (1) and (2):

$$
x(\tau)=\sum_{k=-\infty}^{+\infty}\langle x\rangle_{k}(t) e^{j \omega k \tau}
$$

where, $\tau \in\left[t-T_{0}, t\right]$ :

$$
\langle x\rangle_{k}(t)=\frac{1}{T_{0}} \int_{t-T_{0}}^{t} x(\tau) e^{-j \omega k \tau} d \tau,
$$

where $\langle x\rangle_{k}(t)$ is the $k$ th Fourier coefficient (the $k$ thorder dynamic phasor) of the signal and $k$ denotes the order of the harmonic. Decomposition into dynamic phasors is a linear operation. In applying this transformation to dynamical systems, the following two properties are often used [15]:

$$
\begin{aligned}
& \frac{d\langle x\rangle_{k}(t)}{d t}=\left\langle\frac{d x(t)}{d t}\right\rangle_{k}-j \omega k\langle x\rangle_{k}(t), \\
& \langle u(t) v(t)\rangle_{k}=\sum_{l=-\infty}^{+\infty}\langle u(t)\rangle_{l}\langle v(t)\rangle_{k-1} .
\end{aligned}
$$

Eqs. (2) to (4) are normally used to model a system in the extended-frequency dynamic phasor domain. Then, Eq. (1) is used to reconstruct a waveform in the time-domain. As stated earlier, any desired number of extended-frequency dynamic phasors may be calculated and any desired number may be used in reconstruction. 


\section{MMC modeling using extended-frequency dynamic phasors}

Figure 1 shows the circuit diagram of a simple MMC connected to an ac system. The Kirchhoff's voltage law is applied to the upper- and lower-arm loops and also to the upper- and lower-arm submodules to obtain the following four state equations for the arm inductor currents and submodule capacitor voltages as shown in Eq. (5):

$$
\begin{aligned}
& L \frac{d i_{j}^{u}}{d t}=V_{d c}^{u}-r i_{d c}^{u}-v_{c j}^{u}-R i_{j}^{u}-\left(L_{s}+L_{t f}\right) \frac{d\left(i_{j}^{u}-j_{j}^{l}\right)}{d t} \\
& \quad-R_{s}\left(j_{j}^{u}-j_{j}^{l}\right)-V_{s j}, \\
& L \frac{d i_{j}^{l}}{d t}=V_{d c}^{l}-r i_{d c}^{l} v_{c j}^{l}-R i_{j}-\left(L_{s}+L_{t f}\right) \frac{d\left(i_{j}^{u}-j_{j}^{l}\right)}{d t} \\
& \quad+R_{s}\left(i_{j}^{u}-j_{j}^{l}\right)+V_{s j} \\
& C_{m} \frac{d v_{a v-j}^{u}}{d t}=\frac{n_{j}^{u} i_{j}^{u}}{N} \\
& C_{m} \frac{d v_{a v-j}^{l}}{d t}=\frac{n_{j}^{l} i_{j}^{l}}{N}
\end{aligned}
$$

where $j=a, b, c$, and superscripts $u$ and $l$ denote the upper and the lower arms, respectively. Other variables are defined in the Nomenclature at the end of the paper and are marked in Figure 1 as well. Here, $v_{c j}^{u}, v_{c j}^{l}$, $i_{d c}^{u}$, and $i_{d c}^{l}$ in the equations shown in Eq. (5) can be substituted as follows:

$$
\begin{aligned}
& v_{c j}^{u}=n_{j}^{u} v_{a v-j}^{u}, \quad \text { and } \quad v_{c j}^{l}=n_{j}^{l} v_{a v-j}^{l}, \\
& i_{d c}^{u}=i_{a}^{u}+i_{b}^{u}+i_{c}^{u}, \quad \text { and } \quad i_{d c}^{l}=i_{a}^{l}+i_{b}^{l}+i_{c}^{l} .
\end{aligned}
$$

To avoid complexity, the summation and the difference of the upper and lower inductor currents and capacitor voltages are considered. The resulting state equations are given in Eq. (6):

$$
\begin{aligned}
\frac{d i_{j}^{d}}{d t}= & -\left(\frac{R+2 R_{s}}{L+2 L_{t f}+2 L_{s}}\right) i_{j}^{d} \\
& +\left(\frac{-r i_{d c}^{d}-\left(n_{j}^{s} v_{a v-j}^{d}+n_{j}^{s} v_{a v-j}^{d}\right) / 2-2 V_{s j}}{L+2 L_{t f}+2 L_{s}}\right), \\
\frac{d i_{j}^{s}}{d t}=- & \left(\frac{R}{L}\right) i_{j}^{s} \\
& +\left(\frac{V_{d c}-r i_{d c}^{s}-\left(n_{j}^{s} v_{a v-j}^{s}+n_{j}^{d} v_{a v-j}^{d}\right) / 2}{L}\right) \\
\frac{d v_{a v-j}^{d}}{d t}= & \left(\frac{n_{j}^{s} i_{j}^{d}+n_{j}^{d} i_{j}^{s}}{2 N C_{m}}\right), \\
\frac{d v_{a v-j}^{d}}{d t}= & \left(\frac{n_{j}^{s} i_{j}^{s}+n_{j}^{d} i_{j}^{d}}{2 N C_{m}}\right) \quad j=a, b, c,
\end{aligned}
$$

where superscripts $s$ and $d$ denote the summation and difference, respectively.

In Eq. (6), other than the state variable, the only unknown is $n_{j}$, which is the switching signal for the MMC. Generating switching signal is explained in Section 4.4. From the state equations in Eq. (6), the corresponding dynamic phasor equations are obtained using the basic operations given in Eqs. (2), (3), and (4). The resulting dynamic phasor equations are given in Eq. (7), as shown in Box I, wherein $j$ denotes a phase and $k$ denotes a harmonic order: The difference between the upper- and the lower-arm currents directly

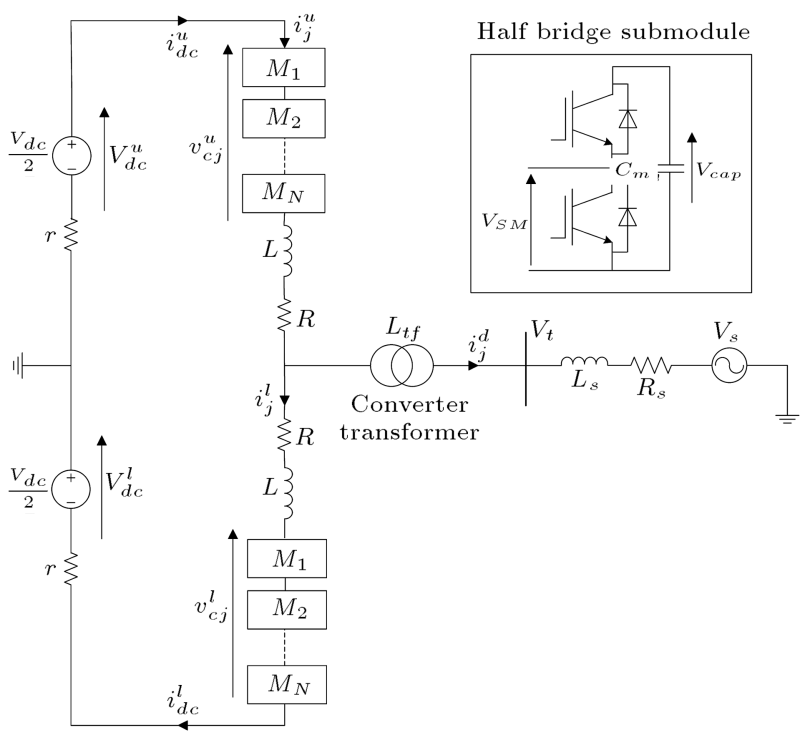

Figure 1. Schematic diagram of an MMC connected to an ac system. 


$$
\begin{aligned}
& \frac{d\left\langle i_{j}^{d}\right\rangle_{k}}{d t}=-j \omega k\left\langle i_{j}^{d}\right\rangle_{k}-\left(\frac{R+2 R_{s}}{L+2 L_{t f}+2 L_{s}}\right)\left\langle i_{j}^{d}\right\rangle_{k} \\
& +\left(\frac{-r\left\langle i_{d c}^{r}\right\rangle_{k}-\left(\sum_{l=-\infty}^{+\infty}\left\langle n_{j}^{s}\right\rangle_{l}\left\langle v_{a c-j}^{d}\right\rangle_{k-l}+\sum_{l=-\infty}^{+\infty}\left\langle n_{j}^{d}\right\rangle_{l}\left\langle v_{a v-j}^{s}\right\rangle_{k-l}\right) / 2-2\left\langle V_{s j}\right\rangle_{k}}{L+2 L_{t f}+{ }_{2} L_{s}}\right), \\
& \frac{d\left\langle i_{j}^{s}\right\rangle_{k}}{d t}=-j \omega k\left\langle i_{j}^{s}\right\rangle_{k}-\left(\frac{R}{L}\right)\left\langle i_{j}^{s}\right\rangle_{k}+\left(\frac{\left\langle V_{d c}\right\rangle_{k}-r\left\langle i_{d c}^{s}\right\rangle_{k}-\left(\sum_{l=-\infty}^{+\infty}\left\langle n_{j}^{s}\right\rangle_{l}\left\langle v_{a v-j}^{s}\right\rangle_{k-l}+\sum_{l=-\infty}^{+\infty}\left\langle n_{j}^{d}\right\rangle_{l}\left\langle v_{a v-j}^{d}\right\rangle_{k-l}\right) / 2}{L}\right), \\
& \frac{d\left\langle v_{a v-j}^{d}\right\rangle_{k}}{d t}=-j \omega k\left\langle v_{a v-j}^{d}\right\rangle_{k}+\left(\frac{\sum_{l=-\infty}^{+\infty}\left\langle n_{j}^{s}\right\rangle_{l}\left\langle i_{j}^{d}\right\rangle_{k-l}+\sum_{l=-\infty}^{+\infty}\left\langle n_{j}^{d}\right\rangle_{l}\left\langle i_{j}^{s}\right\rangle_{k-l}}{2 N C_{m}}\right) \\
& \frac{d\left\langle v_{a v-j}^{s}\right\rangle_{k}}{d t}=-j \omega k\left\langle v_{a v-j}^{s}\right\rangle_{k}+\left(\frac{\sum_{l=-\infty}^{+\infty}\left\langle n_{j}^{s}\right\rangle_{l}\left\langle i_{j}^{s}\right\rangle_{k-l}+\sum_{l=-\infty}^{+\infty}\left\langle n_{j}^{d}\right\rangle_{l}\left\langle i_{j}^{d}\right\rangle_{k-l}}{2 N C_{m}}\right) .
\end{aligned}
$$

gives the line current. Note that $\left\langle i_{j}^{d}\right\rangle$ and $\left\langle v_{a v-j}^{d}\right\rangle$ only contain odd harmonics; likewise, $\left\langle i_{j}^{s}\right\rangle$ and $\left\langle v_{a v-j}^{s}\right\rangle$ only contain even harmonics.

\section{Dynamic phasor modeling of MMC controllers}

In the considered MMC test system, the real power and the RMS terminal voltage are controlled via closedloop control systems shown in Figure 2. As shown, the errors between the reference and the actual variables of interest are fed through Proportional-Integral (PI) controllers that eventually generate commands for the converter's modulation index and phase shift. The converter's output voltage phase-shift is relative to a reference sinewave that is achieved using a PhaseLocked Loop (PLL) unit connected to the Point of Common Coupling (PCC). The controller inputs are the reference values of real power and RMS terminal voltage; the controller outputs are also (quasi-)dc

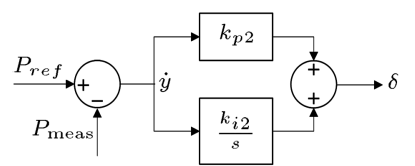

(a)

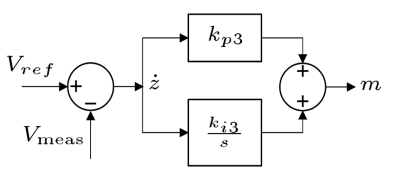

(b)
Figure 2. Schematic diagram of the MMC control system: (a) Real power controller; and (b) Terminal voltage controller. quantities. As such, in their dynamic phasor model, inclusion of harmonics is not necessary.

\subsection{PI controller modeling}

4.1.1. Real power controller

From Figure 2(a), the state equations for the real power controller can be obtained as follows:

$$
\begin{aligned}
& \frac{d y}{d t}=P_{\text {ref }}-P_{\text {meas }}, \\
& \delta=k_{p 2} \frac{d y}{d t}+k_{i 2} y .
\end{aligned}
$$

Real power has primarily only a dc component. Therefore, only one value of $\delta$ is needed (i.e., no harmonicorder model for the PI controller is developed); $P_{r e f}$ is the ordered power and the actual power is determined as follows:

$$
\begin{aligned}
P_{\text {meas }}= & V_{t a} \cdot i_{a}+V_{t b} . i_{b}+V_{t c} \cdot i_{c} . \\
\left\langle P_{\text {meas }}\right\rangle_{k}= & \sum_{l=0}^{\infty}\left\langle V_{t a}\right\rangle_{l}\left\langle i_{a}\right\rangle_{k-l}+\sum_{l=0}^{\infty}\left\langle V_{t b}\right\rangle_{l}\left\langle i_{b}\right\rangle_{k-l} \\
& +\sum_{l=0}^{\infty}\left\langle V_{t c}\right\rangle_{l}\left\langle i_{c}\right\rangle_{k-l}, \\
\left\langle P_{\text {meas }}\right\rangle_{0}= & \sum_{l=0}^{\infty}\left\langle V_{t a}\right\rangle_{l}\left\langle i_{a}\right\rangle_{-l}+\left\langle V_{t b}\right\rangle_{l}\left\langle i_{b}\right\rangle_{-l}+\left\langle V_{t c}\right\rangle_{l}\left\langle i_{c}\right\rangle_{-l},
\end{aligned}
$$




\subsubsection{Terminal RMS voltage controller model}

The following state equations of the voltage controller are developed for the control block diagram shown in Figure 2(b):

$$
\begin{aligned}
& \frac{d z}{d t}=V_{\text {ref }}-V_{\text {meas }}, \\
& m=k_{p 3} \frac{d z}{d t}+k_{i 3} z .
\end{aligned}
$$

The terminal voltage is a staircase waveform obtained using stacking of an appropriate number of submodules; due to its quarter-cycle symmetry, it contains only odd harmonics. The controller input, $V_{\text {meas }}$, is the line-to-line RMS value. The real and imaginary values of the terminal voltage for each harmonic are known, and the basic RMS equation and the corresponding dynamic phasor equation are given as follows:

$$
\begin{aligned}
& V_{\text {meas }}=\sqrt{\frac{3}{2}} \cdot V_{\text {tapeak }}, \\
& \left\langle V_{\text {meas }}\right\rangle_{k}=\sqrt{\frac{3}{2}} \cdot 2 \sqrt{\operatorname{Re}\left(\left\langle V_{t a}\right\rangle_{k}\right)^{2}+\operatorname{Im}\left(\left\langle V_{t a}\right\rangle_{l}\right)^{2}} .
\end{aligned}
$$

The dynamic phasor form of Eq. (10) is shown in Eq. (12). In Eq. (12), $V_{\text {meas }}$ represents the magnitude of $k$ th harmonic that is a $d c$ value. Therefore, the output modulation indices for each harmonic are also dc values:

$$
\begin{aligned}
& \left\langle\frac{d z}{d t}\right\rangle_{k}=\left\langle V_{r e f}\right\rangle_{k}-\left\langle V_{\text {meas }}\right\rangle_{k}, \\
& \langle m\rangle_{k}=k_{p 3}\left\langle\frac{d z}{d t}\right\rangle_{k}+k_{i 3}\langle z\rangle_{k} .
\end{aligned}
$$

Using Eq. (12) for each odd harmonic, the modulation index values corresponding to each harmonic can be obtained. By using the modulation index values in the switching signal, the terminal voltage can be regulated. To find the reference values for each harmonic, the nearest level control (explained in Section 4.3) is used.

\subsection{Modeling of the phase locked loop}

A PLL is a feedback control system that rapidly tracks the phase angle of a given sinusoidal voltage. A dynamic phasor model of the PLL is developed in [9] and its block diagram is shown in Figure 3. This model is directly used in this study as well. Here, $\omega_{0}$ is the nominal system frequency (i.e., $2 \pi 60 \mathrm{rad} / \mathrm{s}$ in a $60-\mathrm{Hz}$ system).

From Figure 3, the following state equations are obtained for the PLL:

$$
\begin{aligned}
& \frac{d x}{d t}=\angle V_{t}-\theta, \\
& \frac{d \theta}{d t}=k_{p 1}\left(\angle V_{t}-\theta\right)+k_{i 1} x .
\end{aligned}
$$

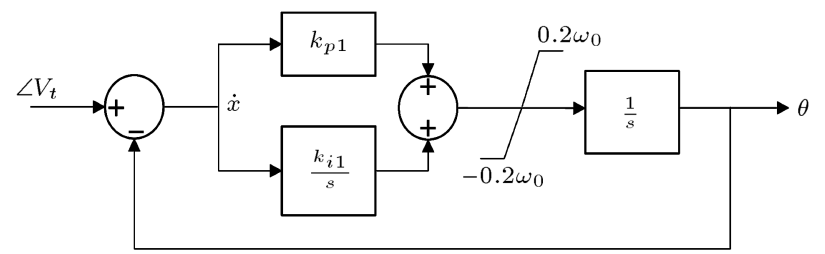

Figure 3. Dynamic phasor model of a Phase Locked Loop (PLL).

For conversion to the dynamic phasor domain, only the $k=0$ component of the PLL state equations is considered. The output $\theta$ is used to generate the switching signals for the converter, as explained in Section 4.4 .

\subsection{Nearest level control and reference voltage harmonics}

The MMC control system determines the fundamental value of $V_{\text {ref }}$ and from that, a staircase voltage waveform closely resembling a sinewave with such magnitude must be generated. To generate the staircase waveform, a modulation technique is needed. There are a number of high-frequency PWM techniques that result in high switching losses. Therefore, in this paper, a low-frequency modulation technique known as the Nearest Level Control (NLC) is used [7]. For this method, the nominal value of the submodule capacitor voltage is needed, which can be calculated as follows:

$$
V_{c a p}=\frac{V_{d c}}{N} .
$$

To obtain the staircase waveform, the following formulae are used:

For even values of $N$ :

$$
V_{\text {out }}= \begin{cases}n V_{\text {cap }} ; & V_{\text {ref }}<\left(n+\frac{1}{2}\right) V_{\text {cap }} \\ (n+1) V_{\text {cap }} ; & V_{\text {ref }}>\left(n+\frac{1}{2}\right) V_{\text {cap }}\end{cases}
$$

where $0 \leq n \leq\left(\frac{N}{2}\right)$.

For odd values of $N$ :

$$
V_{\text {out }}= \begin{cases}\left(n-\frac{1}{2}\right) V_{\text {cap }} ; & V_{\text {ref }}<n V_{\text {cap }} \\ \left(n+\frac{1}{2}\right) V_{\text {cap }} ; & V_{\text {ref }}>n V_{\text {cap }}\end{cases}
$$

where:

$$
0 \leq n \leq\left(\frac{N-1}{2}\right)
$$

For the odd and even numbers of submodules per arm, the staircase waveform is different. However, the final number of the levels in the output staircase waveform is $N+1$.

Figure 4 shows the staircase waveform for a half cycle when the number of submodules is 8 . Therefore, 


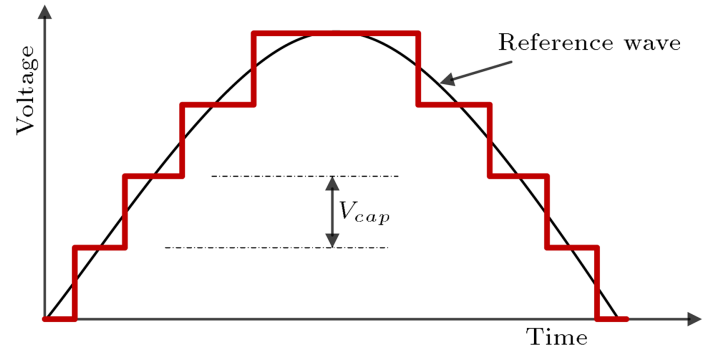

Figure 4. Staircase waveform (positive half-cycle shown) for $N=8$.

the number of levels seen in the output waveform is 9 for both positive and negative half cycles. The switching instants between levels correspond to the nearest level angles. By using Eq. (2) for each value of $k$, the $k$ th Fourier coefficient can be calculated, which can then be converted to line-to-line RMS and given as the reference voltage for each harmonic in the voltage controller.

\subsection{Generation of switching signals}

To generate the switching signals for the upper- and lower-arms, the modulation index and the phase angle from the controller and PLL angle are required. The upper- and lower-arm switching signals for phase ' $a$ ' are given in Eq. (16):

$$
\begin{aligned}
& n_{a}^{u}=\frac{N}{2}-\frac{N}{2} \sum_{k=1}^{\infty}\langle m\rangle_{k} \sin \left(k \omega t+k \theta+\delta_{k}\right), \\
& n_{a}^{l}=\frac{N}{2}+\frac{N}{2} \sum_{k=1}^{\infty}\langle m\rangle_{k} \sin \left(k \omega t+k \theta+\delta_{k}\right),
\end{aligned}
$$

where $k$ is an odd number. For phases ' $b$ ' and ' $c$ ', phase shifts of $120^{\circ}$ and $240^{\circ}$ need to be added, respectively. Since the difference and the sum of the switching signals are used in Eqs. (6) and (7), the sum and difference of the switching signals in Eq. (16) need to be obtained as follows:

$$
n_{a}^{d}=-N \sum_{k=1}^{\infty}\langle m\rangle_{k} \sin \left(k \omega t+k \theta+\delta_{k}\right)
$$

$$
n_{a}^{s}=N
$$

As can be seen from Eq. (17), the sum of the switching signals is always a constant and it is equal to the number of submodules per arm of the MMC. The only time-variant part is the difference of the switching signals. Therefore, using the basic form in Eq. (2), the dynamic phasor representation of $n_{j}^{d}$ can be found as given in Eq. (18):

$$
\begin{aligned}
n_{a}^{d}= & -N\left\{\langle m\rangle_{1} \sin \left(\omega t+\theta+\delta_{1}\right)+\langle m\rangle_{3} \sin (3 \omega t\right. \\
& \left.\left.+3 \theta+\delta_{3}\right)+\langle m\rangle_{5} \sin \left(5 \omega t+5 \theta+\delta_{5}\right)+\ldots\right\} \\
\left\langle n_{a}^{d}\right\rangle_{k}= & -\frac{N\langle m\rangle_{k}}{2 j} e^{j\left(k \theta+\delta_{k}\right)}=\frac{j N\langle m\rangle_{k}}{2} e^{j\left(k \theta+\delta_{k}\right)} .
\end{aligned}
$$

Similarly, the switching signals corresponding to other two phases can be obtained. For odd values of $k$, the $k$ th harmonic of switching signal can be obtained.

\section{Model validation}

A detailed switching model of the MMC test system is developed using PSCAD/EMTDC electromagnetic transient simulation program. EMT simulators such as PSCAD/EMTDC are widely used to study fast transients in power systems with embedded power electronics. Due to their detailed component models and accurate solution methods, EMT simulation results are generally accepted to be close representations of the real phenomena, and as such it is common to use them as benchmarks for validation of other simulators. The parameters of the simulated circuit are given in Table 1.

To show the details of the output staircase waveform, the number of submodules per arm $(N)$ is selected as 5 . In addition, to minimize the burden on modeling, large capacitors $(5000 \mu \mathrm{F})$ are selected so that the effect of circulation current becomes minimal.

Table 1. Power system parameters.

\begin{tabular}{ll}
\hline \multicolumn{1}{c}{ Parameters } & \multicolumn{1}{c}{ Value } \\
\hline AC system rated voltage & $290 \mathrm{kV}$ \\
Short circuit ratio & $4.0 \angle 80^{\circ}$ \\
Rated power & $500 \mathrm{MW}$ \\
Rated dc voltage, internal resistance & $500 \mathrm{kV}, 0.001 \Omega$ \\
Converter transformer turns ratio & $290: 290$ \\
Converter transformer impedance & $0.05 \mathrm{pu}, 700 \mathrm{MVA}$ \\
Arm inductor & $1 \mathrm{mH}$ \\
Submodule capacitor & $5000 \mu \mathrm{F}$ \\
Nominal voltage of submodule capacitor & $100 \mathrm{kV}$ \\
\hline
\end{tabular}


The parameters of the PI controllers are given in Table 2. The values of proportional $\left(k_{p}\right)$ and integral gain $\left(k_{i}\right)$ are selected based upon trial and error.

In the following sections, all PSCAD/EMTDC results are obtained with a $50 \mu$ s time-step and all dynamic phasor results are obtained with a $100 \mu \mathrm{s}$ time-step.

\subsection{Model validation for the fundamental frequency}

In Figure 5, the comparison of results from the dynamic phasor and EMT models is shown only for the fundamental component. Although the EMT model includes all harmonics, the fundamental components of the shown waveforms are extracted using a standard FFT block. The dynamic phasor model in this set of simulations only includes the fundamental component. The results clearly show that the waveforms are well matched.

\subsection{Model validation including harmonics}

Figure 6 shows the comparison of the EMT results and the dynamic phasor representation of harmonics up to the 17th harmonic. If more harmonics are added to the dynamic phasor model, it will produce much more accurate results that will eventually converge more closely at the EMT traces. However, even with the

Table 2. Controller parameters.

\begin{tabular}{lcc}
\hline \multicolumn{1}{c}{ Controllers } & $\boldsymbol{k}_{\boldsymbol{p}}$ & $\boldsymbol{k}_{\boldsymbol{i}}$ \\
\hline PLL & 50 & 900 \\
Real power controller & 0.04 & 0.25 \\
Terminal voltage controller & 0.005 & 50 \\
\hline
\end{tabular}
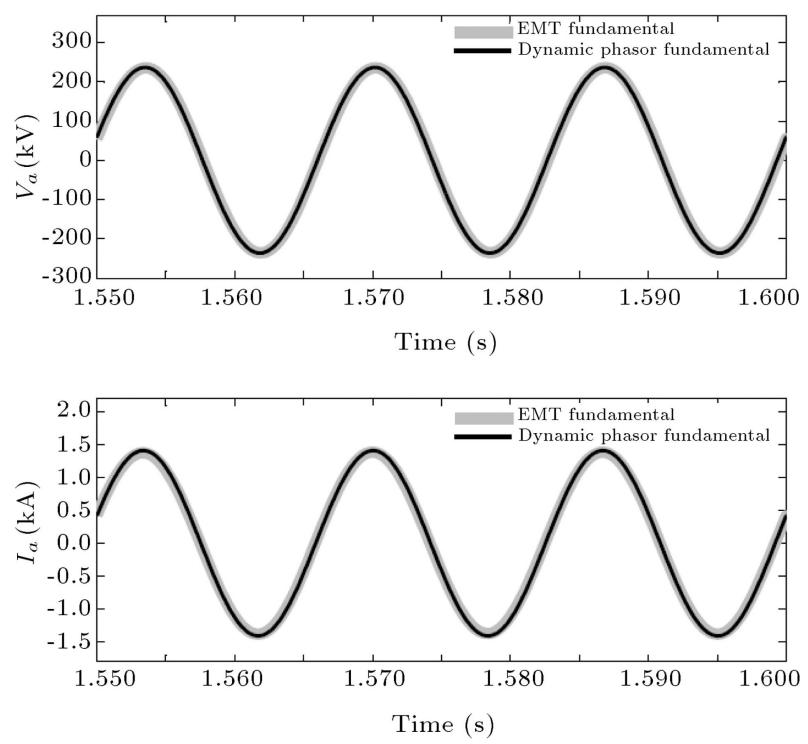

Figure 5. Comparison of steady-state results (fundamental only).
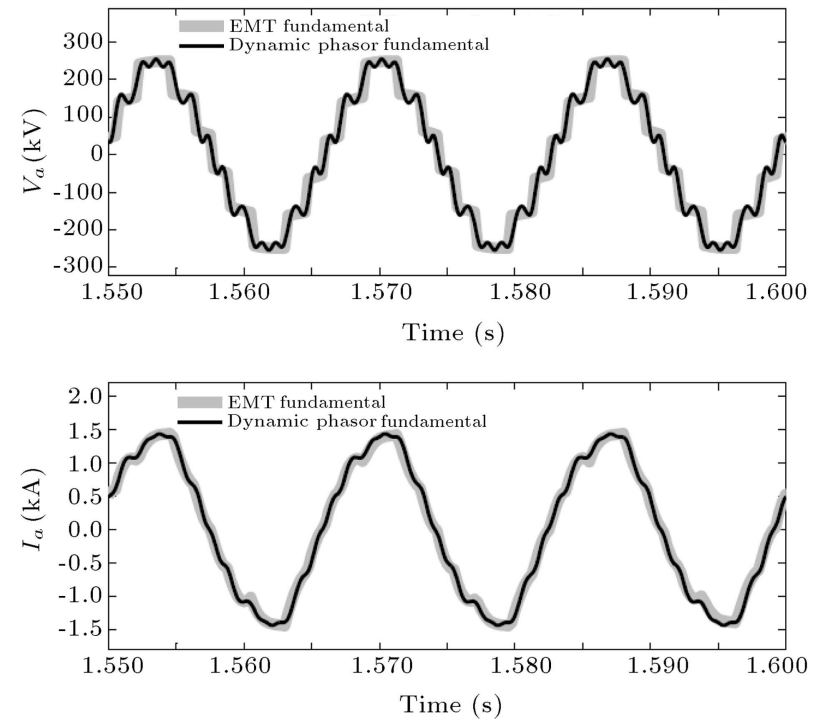

Figure 6. Comparison of steady-state results with inclusion of harmonics.

harmonics up to 17 th, the waveforms show a very close similarity with the EMT simulation results.

\subsection{Validation with a step change in terminal voltage reference}

To test the model accuracy further, a step decrease is applied to the terminal voltage reference from 1 p.u. to 0.75 p.u. at $2 \mathrm{~s}$. The smooth terminal voltage transition obtained through the voltage controller in dynamic phasor model is shown in Figure 7.

Figure 8 shows the comparison of fundamental results from the EMT model and the dynamic phasor model for the step change in voltage.

It can be seen that results from both models closely match at transient (around $2 \mathrm{~s}$ ) and in steady state. The slow delay observed in the current waveform can be the effect of the voltage controller.

Figure 9 shows the comparison of waveforms with the same step change in the voltage reference with inclusion of the harmonics. The EMT model has all the harmonics and the dynamic phasor model has harmonics up to the 17 th. For the terminal voltage change from 1 p.u to 0.75 p.u., the levels in output

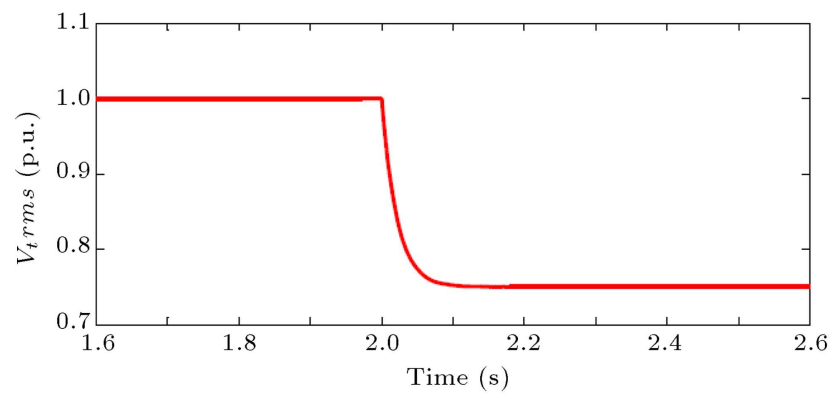

Figure 7. Response to a step change in the terminal voltage reference. 

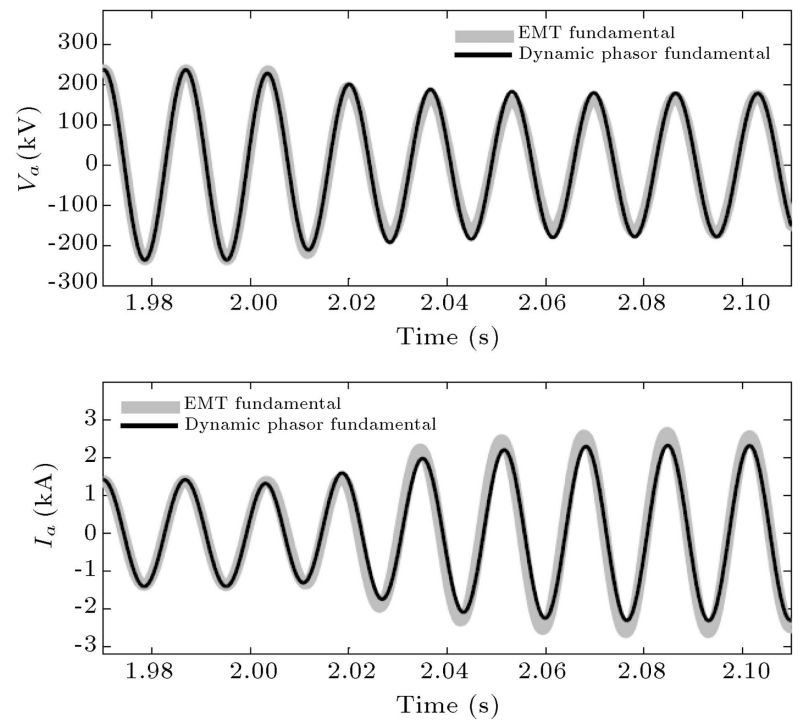

Figure 8. Comparison of the responses to a step change in the voltage reference (fundamental only).
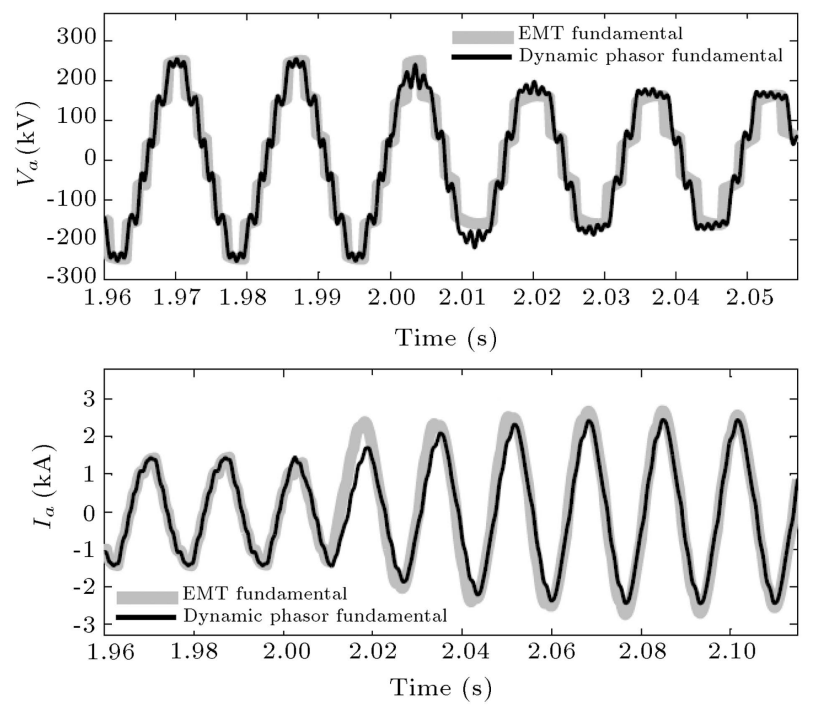

Figure 9. Comparison of the responses to a step change in the voltage reference (including harmonics).

voltage changed from 6 to 4 . The line current increased to maintain constant power flow at $500 \mathrm{MW}$.

\subsection{Model validation with a step change in the real power reference}

A step change in the real power order from $300 \mathrm{MW}$ to $500 \mathrm{MW}$ is applied to both models at $2 \mathrm{~s}$, and the RMS power variation obtained from the dynamic phasor model is shown in Figure 10.

Figure 11 shows the variation in the phase ' $a$ ' voltage and current (fundamental component only) waveforms in response to the power order change. It is observed that the dynamic phasor model closely follows the step change compared to the EMT model. Note that similar to the previous case, the fundamental component of the EMT solution is obtained

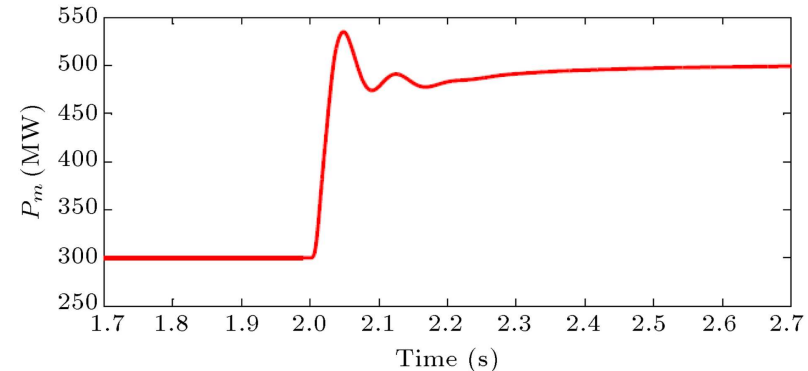

Figure 10. Response to a step change in terminal real power.
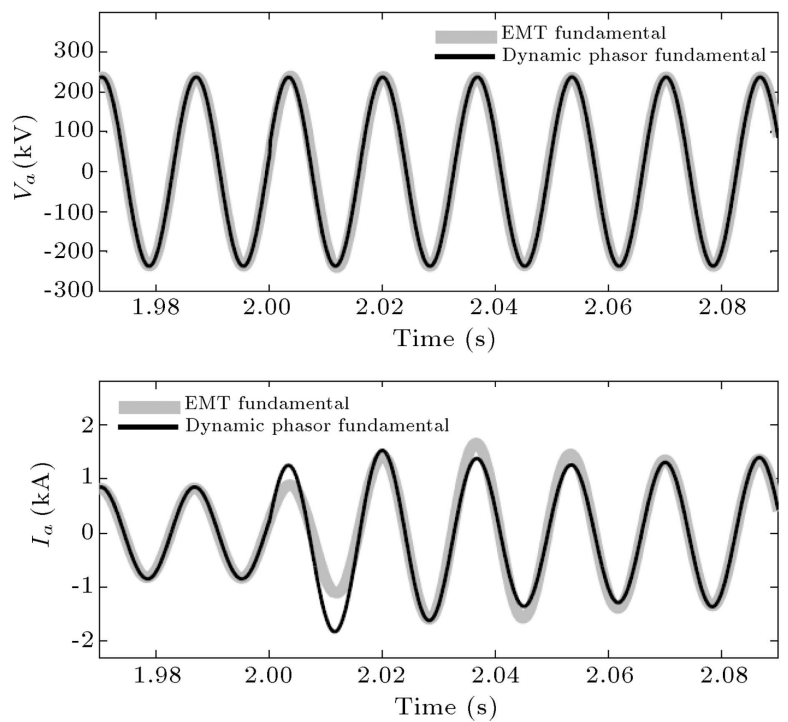

Figure 11. Comparison of the responses to a step change in the power reference (fundamental only).

using a standard FFT block in PSCAD/EMTDC from the original response waveform including all harmonics.

Figure 12 shows the phase ' $a$ ' voltage and current with inclusion of harmonics for a step change in the power order. It can be observed that the power order change does not affect the ac voltage in a noticeable way and the change is only observed in the line current.

\subsection{Analysis of the simulation error}

It is straightforward to note that a dynamic phasor based model can be made more accurate if a larger number of harmonics are included in the model. This section shows how doing so affects the accuracy of a simulated waveform. As an example, the dynamic phasor-based representation of the terminal voltage waveform with an increasing number of odd harmonic is compared against the actual staircase waveform. The RMS error between the dynamic phasor voltage waveforms and the actual staircase waveform is plotted in Figure 13 as a function of the number of harmonic components included.

It is observed from Figure 13 that inclusion of 

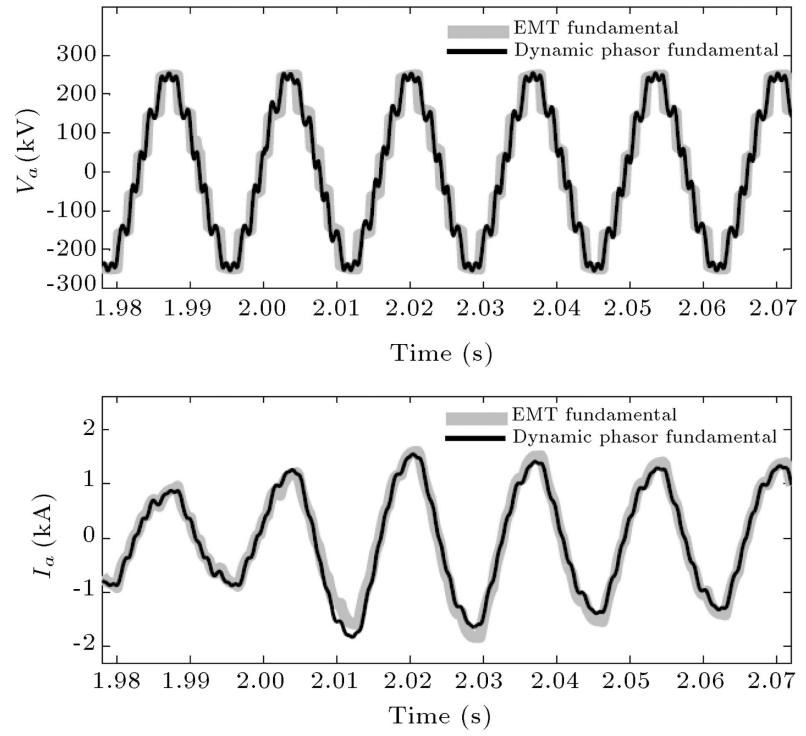

Figure 12. Comparison of the responses to a step change in the power reference (including harmonics).

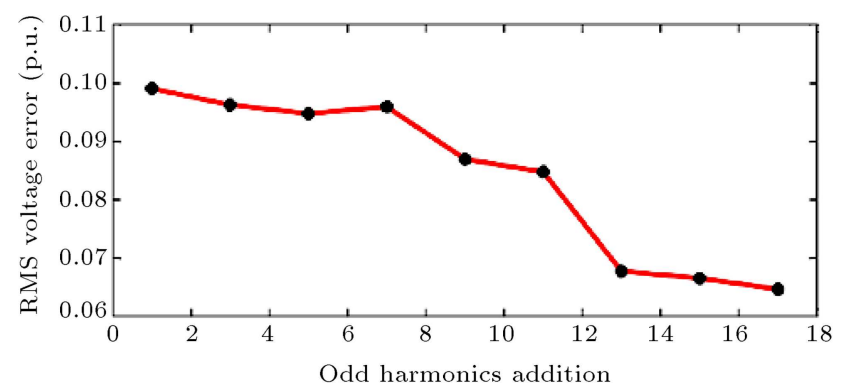

Figure 13. Model's error as a function of harmonics included.

every odd harmonic (up to the 17th as shown) leads to a reduction in RMS error. In general, the observation made in Figure 13 holds true for models developed using dynamic phasors. The model accuracy improves when a larger number of constituent harmonics are included; however, it must also be noted that most power systems dampen high-frequency harmonics due to their inductive nature. Therefore, as the harmonic frequencies increase, their expected contribution is expected to decrease. As such, inclusion of highfrequency harmonics may not have marked contributions to the accuracy of the results beyond a certain point. This is also evident in Figure 13, as the RMS error appears to reach a flat level beyond the 13th harmonic.

Note that in practical MMC configurations, a much larger number of submodules are normally used. Therefore, the harmonic contents of the actual voltage waveform will be much reduced compared to the case considered in this paper. As such, it is expected that for simulations of practical MMC systems, a much smaller number of harmonics need to be included to achieve high levels of accuracy.

\section{Conclusion}

An extended-frequency dynamic phasor model of an MMC was presented and validated against a detailed EMT model in PSCAD/EMTDC. The fundamentalfrequency results of the dynamic phasor model closely matched the fundamental frequency response of EMT simulations. By adding more harmonics to the dynamic phasor model, further accuracy was achieved. It was also shown that a dynamic phasor model, even with consideration of an extended number of harmonics, is executable with a much larger time step than required in an EMT simulation, thereby offering significant computational advantages.

\section{Acknowledgements}

This research was funded by the Natural Sciences and Engineering Research Council (NSERC) of Canada, MITACS Accelerate, and Manitoba Hydro.

\section{Nomenclature}

$\begin{array}{ll}i_{j} & \text { Arm currents } \\ v_{a v-j} & \text { Submodule average voltages } \\ n_{j} & \text { Switching functions } \\ i_{d c} & \text { DC currents } \\ v_{c j} & \text { Sum of submodule voltages in the arm } \\ V_{d c}, r & \text { DC link voltage and DC source internal } \\ \omega=2 \pi f & \text { resistance } \\ N & \text { Angular frequency } \\ C_{m} & \text { Sumber of submodules per arm } \\ L_{t f} & \text { Converter transformer inductance } \\ R, L & \text { Arm resistance and arm inductance } \\ R_{s}, L_{s} & \text { AC network Thévenin resistance and } \\ & \text { inductance } \\ V_{s j} & \text { AC network source voltage }\end{array}$

\section{References}

1. Dorn, J., Huang, H. and Retzmann, D. "A new multilevel voltage-sourced converter topology for HVDC applications", B4-304-2008, CIGRE Meeting, 1, Paris, France (2008).

2. Nami, A., Liang, J., Dijkhuizen, F. and Demetriades, G.D. "Modular multilevel converters for HVDC applications: review on converter cells and functionalities", IEEE Trans. Power Electron., 1(30), pp. 18-36 (2015).

3. Trainer, D.R., Davidson, C.C., Oates, C.D.M., Macleod, N.M., Critchley, D.R. and Crookes, R.W. "A new hybrid voltage-sourced converter for HVDC power transmission", B4-111-2010, CIGRE Meeting, 1, Paris, France (2010). 
4. Jacobson, B., Karlsson, P., Asplund, G., Harnefors, L. and Jonsson, T. "VSC-HVDC transmission with cascaded two-level converters", B4-110-2010, CIGRE Meeting, 1, Paris, France (2010).

5. Saeedifard, M. and Iravani, R. "Dynamic performance of a modular multilevel back-to-back HVDC system", IEEE Trans. Power Del., 4(25), pp. 2903-2912 (2010).

6. Saeedifard, M., Nikkhajoei, H., Iravani, R. and Bakhshai, A. "A space vector modulation approach for a multi module HVDC converter system", IEEE Trans. Power Del., 3(22), pp. 1643-1654 (2007).

7. Meshram, P.M. and Borghate, V.B. "A simplified nearest level control (NLC) voltage balancing method for modular multilevel converter (MMC)", IEEE Trans. on Power Electron., 1(30), pp. 450-462 (2015).

8. Chiniforoosh, S., Jatskevich, J., Yazdani, A., Sood, V.K., Dinavahi, V., Martinez, J.A. and Ramirez, A. "Definitions and applications of dynamic average models for analysis of power systems", IEEE Trans. Power Del., 4(25), pp. 2655-2669 (2010).

9. Daryabak, M., Filizadeh, S., Jatskevich, J., Davoudi, A., Saeedifard, M., Soodm V.K., Martinez, J.A., Aliprantis, D., Cano, J. and Mehrizi-Sani, A. "Modeling of LCC-HVDC systems using dynamic phasors", IEEE Trans. on Power Del., 4(29), pp. 1989-1998 (2014).

10. Yao, W., Wen, J. and Cheng, S. "Modeling and simulation of VSC-HVDC with dynamic phasors", Electric Utility Deregulation and Restructuring and Power Technologies (DRPT 2008), 1, pp. 1416-1421 (2008).

11. Chandrasekar, S. and Gokaraju, R. "Dynamic phasor modeling of type 3 DFIG wind generators (including SSCI phenomenon) for short-circuit calculations", IEEE Trans. on Power Del., 2(30), pp. 887-897 (2015).

12. Deore, S.R., Darji, P.B. and Kulkarni, A.M. "Dynamic phasor modeling of modular multi-level converters", IEEE International Conference on Ind. and Inf. Sys. (ICIIS), 1, pp. 1-6 (2012).
13. Darji, P.B. and Kulkarni, A.M. "Dynamic performance of a modular multi-level converter based HVDC terminal under unbalanced AC grid conditions", 10th Int. Conf. AC-DC Power Transmiss., 1, pp. 1-6 (2012).

14. Jovcic, D. and Jamshidifar, A. "Phasor model of modular multilevel converter with circulating current suppression control", IEEE Trans. on Power Del., 4(30), pp. 1889-1897 (2015).

15. Sanders, S.R., Noworolski, J.M., Liu, X.Z. and Verghese, G.C. "Generalized averaging method for power conversion circuits", IEEE Trans. Power Electron., 2(6), pp. 251-259 (1991).

\section{Biographies}

Shailajah Rajesvaran graduated with a BSc (Hons.) from the University of Peradeniya in Peradeniya, Sri Lanka, in 2012. Since Jan. 2014, she has been pursuing an MSc degree in the Department of Electrical and Computer Engineering at the University of Manitoba. Prior to joining the University of Manitoba, she was an Electrical Design Engineer with Hairu Engineering Consultancy Company (Pvt.) Ltd in Sri Lanka.

Ms. Rajesvaran's research interests are in power electronic applications in power systems, and modelling and simulation of advanced power converters.

Shaahin Filizadeh received his BSc and MSc degrees in Electrical Engineering from Sharif University of Technology in Tehran, Iran, in 1996 and 1998, respectively. He obtained his $\mathrm{PhD}$ from the University of Manitoba, Canada, in 2004. He is currently a professor with the Department of Electrical and Computer Engineering at the University of Manitoba, where he is also Associate Head (graduate programs).

His areas of interest include electromagnetic transient simulation, nonlinear optimization, and power electronic applications in power systems and vehicle propulsion. Dr. Filizadeh is a senior member of the IEEE and a registered professional engineer in the Province of Manitoba. 